\title{
白杨素衍生物的合成及体外抗弓形虫活性研究
}

\author{
尚凡凡金澜张海滨朴莲荀* 全哲山* \\ (延边大学药学院 延吉 133002)
}

\begin{abstract}
摘要 设计并合成了 18 个未见报道的白杨素类衍生物, 采用噻唑蓝(MTT)比色法对所有化合物进行体外抗弓形虫活性 测定, 其化学结构均经过 ${ }^{1} \mathrm{H} \mathrm{NMR},{ }^{13} \mathrm{C} \mathrm{NMR}$ 以及 HRMS 的联合确证. 结果显示, 7-甲氧基- 8 -( $N, N$-双(二乙羟基)-磺酰胺 基)-白杨素(D2)的选择性指数为 1.76, 其抗弓形虫活性明显强于先导化合物白杨素及阳性对照药磺胺嘧啶, 具有深入 的研究价值.
\end{abstract}

关键词 白杨素类衍生物; 合成; 抗弓形虫活性; 体外; 选择性指数

\section{Synthesis and Anti-toxoplasma Activity in Vitro of Chrysin Derivatives}

\author{
Shang, Fanfan Jin, Lan Zhang, Haibin Piao, Lianxun* Quan, Zheshan* \\ (College of Pharmacy, Yanbian University, Yanji 133002)
}

\begin{abstract}
In this paper, 18 novel chrysin derivatives were designed and synthesized. All target compounds were tested for anti-Toxoplasma activity by methyl thiazolyl tetrazolium (MTT) colorimetric method. The chemical structures were characterized by ${ }^{1} \mathrm{H}$ NMR, ${ }^{13} \mathrm{C}$ NMR and HRMS spectra. The selectivity index of 5-hydroxy-7-methoxy-4-oxo- $N, 2$-diphenyl- $4 H$-chromene-8-sulfonamide (D2) was 1.76, indicating that the anti-toxoplasma activity of compound D2 was significantly higher than that of the lead compound chrysin and the positive control drug sulfadiazine.
\end{abstract}

Keywords chrysin derivatives; synthesis; toxoplasma activity; in vitro; selectivity index

弓形虫(Toxoplasma gondii) 是专性细胞内寄生虫 ${ }^{[1]}$, 其宿主种类繁多, 包括人类、牲畜、海洋哺乳动物等, 可 引起人畜共患的弓形虫病 ${ }^{[2]}$. 免疫功能正常的人感染后 无明显症状; 可免疫功能不全或者低下者感染后可导致 广泛的病理损害, 严重时甚至可导致死亡; 孕妇感染后 可造成流产、早产、死胎、畸胎等 ${ }^{[3]}$. 弓形虫还可感染 猪、牛、羊等家畜, 在狗、猫、兔等伴侣动物中感染较 为普遍，在给畜牧业带来巨大经济损失的同时，为食品 安全和人类健康也埋下了巨大隐患 ${ }^{[4]}$. 目前, 弓形虫病 的主要治疗手段仍旧是药物治疗, 抗弓形虫药物合理、 正确的使用虽然取得了一定的疗效, 但也有不少问题值 得关注, 例如, 抗弓形虫药物在使用过程中常伴随着一 些不良反应, 如过敏、骨髓抑制以及肝肾并发症风险增 加等 ${ }^{[5]}$, 并且所有的药物都不适用于孕妇 ${ }^{[6]}$. 因此, 开发 具有高效、低毒的抗弓形虫药物显得尤为迫切.

白杨素是一种从紫葳科植物木蝴蝶中提取的黄酩 类化合物, 广泛存在于蜂蜜和蜂胶中, 具有诸多药理活
性 ${ }^{[7,8]}$, 如抗氧化 ${ }^{[9]}$ 、抗病毒 ${ }^{[10]}$ 、降血糖 ${ }^{[11]}$ 、抗焦虑 ${ }^{[12]}$ 等, 此外, 白杨素亦被报道具有抗弓形虫活性 ${ }^{[13]}$. 磺胺 类药物(Sulfonamides) 是一类广谱的抗微生物药物, 其 化学结构与弓形虫叶酸代谢中的对氨基苯甲酸类似, 可 竞争性的结合二氢叶酸合成酶从而抑制其活性，导致叶 酸代谢异常，达到抑制或杀灭弓形虫的作用 ${ }^{[14]}$. 基于上 述研究发现, 设想在白杨素化合物 C-8 位引入一系列磺 酰胺类基团, 期望得到抗弓形虫活性更好的新型白杨素 衍生物(图 1).

\section{1 结果与讨论}

\section{1 目标化合物的合成}

目标化合物的合成路线如 Scheme 1 所示, 以白杨 素为起始原料, 用碘甲烷对 C-7 位的酚羟基进行甲基化 反应, 得到化合物 $\mathbf{1}^{[16]}$. 化合物 $\mathbf{1}$ 再与氯磺酸进行磺酸 化反应, 得到在 C-8 位含氯磺酰基的甲基化白杨素

\footnotetext{
* Corresponding authors. E-mail: zsquan@ybu.edu.cn; 1xpiao@ybu.edu.cn Received January 11, 2019; revised February 28, 2019; published online March 21, 2019.

Project supported by the National Natural Science Foundation of China (Nos. 21662036, 81660344) 国家自然科学基金(Nos. 21662036, 81660344)资助项目.
} 


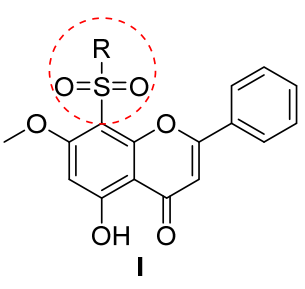

图 1 含磺酰基具有体外抗弓形虫作用的化合物

Figure 1 Compound containing sulfonyl group for anti-toxoplasmosis in vitro

$(2)^{[17]}$. 化合物 2 以二氯甲烷为溶剂, 与各种苄胺类、苯 胺类、脂肪胺类以及杂环类化合物于 $-10{ }^{\circ} \mathrm{C}$ 条件下反 应 $^{[17]}$, 得到目标化合物 $\mathbf{A 1} \sim \mathbf{A 5} 、 \mathbf{B 1} \sim \mathbf{B} 7 、 \mathbf{C 1} \sim \mathbf{C 2}$ 、

D1 D4, 总收率 53.4\% 72.4\%.

\section{2 生物学活性分析}

采用噻唑蓝(MTT)比色法对所有化合物在不同浓度 下 $(500 \sim 0.01 \mu \mathrm{mol} / \mathrm{L})$ 对宿主细胞(RAW 264.7 细胞)的细 胞毒性和体外抗速殖子(T. gondii, RH Strain)活性进行测 试. 根据不同浓度下的抑制率, 计算各个化合物对弓形
虫感染的 RAW 264.7 细胞的 $\mathrm{IC}_{50}$ 值(抑制 $50 \%$ 弓形虫感 染的 RAW 264.7 细胞生长的药物浓度值)与 RAW 264.7 细胞的 $\mathrm{CC}_{50}$ 值(抑制 $50 \%$ 细胞生长的药物浓度值)以及 选择性指数(SI), 选择性指数(SI) 指的是药物对正常 RAW 264.7 细胞的半数抑制浓度与对弓形虫感染 RAW 264.7 细胞的半数抑制浓度之间的比值, 此数值的大小 可以间接反映药物对细胞感染率的影响 ${ }^{[15]}$.

本实验设计并合成了 4 个系列 $(\mathbf{A 1} \sim \mathbf{A 5}, \mathbf{B 1} \sim \mathbf{B} 7$, C1 C 2 D1 D D)共 18 个未见报道的白杨素衍生物. 在 体外实验中, 不同受试化合物的抗弓形虫活性我们用选 择性指数(SI)来表达 ${ }^{[15]}$. 由以上实验数据可知先导物白 杨素的 SI 值(0.82)略小于阳性对照药磺胺嘧啶的 SI 值 (0.91), 这表明白杨素具有一定的抗弓形虫活性, 但其 疗效略低于磺胺嘧啶. 而在对其进行结构修饰后的这些 衍生物中, 所有化合物的体外抗速殖子能力 $\left(\mathrm{IC}_{50}\right.$ in $T$. gondii)与阳性对照药相比均有显著的提升, 其中, 14 个 化合物的抗速殖子能力优于先导物白杨素.

化合物的构效关系: 化合物 $\mathbf{A}$ 系列为引入不同取代 的苯胺基团, 所有化合物均有着很强的抗速殖子能力,<smiles>[R]c1cccc(NS(=O)(=O)c2c(OC)cc(O)c3c(=O)cc(-c4ccccc4)oc23)c1</smiles>

A1 A5

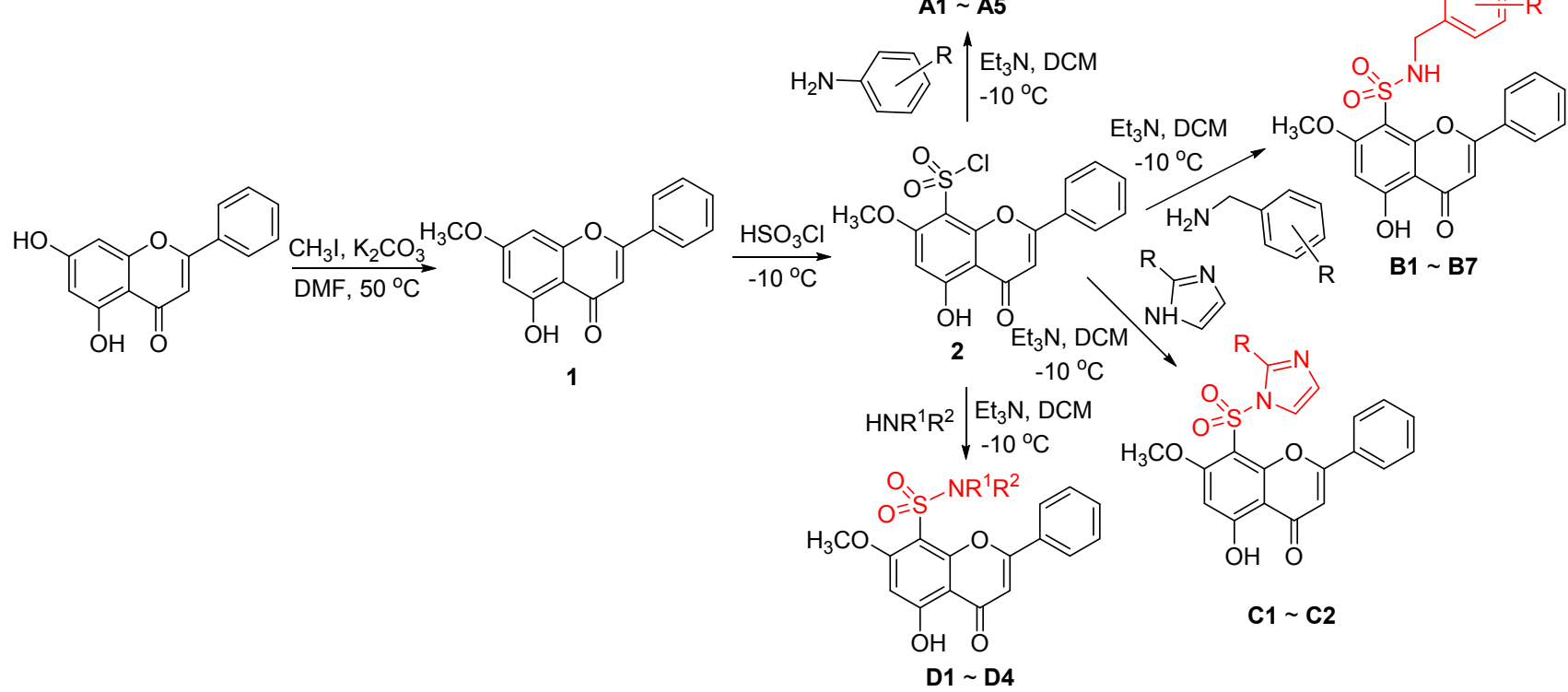
A1: $\mathrm{R}=4-\mathrm{H}$
A2: $\mathrm{R}=4-\mathrm{Cl}$
A3: $\mathrm{R}=3,4-\mathrm{Cl}_{2}$
A4: $\mathrm{R}=4-\mathrm{Br}$
A5: $\mathrm{R}=4-\mathrm{CH}_{3}$
B1: $\mathrm{R}=4-\mathrm{H}$
B2: $\mathrm{R}=4-\mathrm{F}$
B3: $\mathrm{R}=2-\mathrm{Cl}$
B4: $\mathrm{R}=4-\mathrm{Cl}$
B5: $\mathrm{R}=2-\mathrm{Br}$
B6: $\mathrm{R}=4-\mathrm{Br}$
B7: $\mathrm{R}=4-\mathrm{OCH}_{3}$
C1: $\mathrm{R}=\mathrm{H}$
C2: $\mathrm{R}=\mathrm{CH}_{3}$
D2: $\mathrm{R}^{1} \mathrm{R}^{2} \mathrm{~N}=-\hat{\mathrm{N}} \mathrm{NH}$
D4: $\mathrm{R}^{1} \mathrm{R}^{2} \mathrm{~N}=\xi_{\xi} \mathrm{H} \sim \mathrm{OH}$

图式 1 目标化合物的合成路线

Scheme 1 Synthesis of target compounds 
表 1 化合物的体外抗弓形虫活性数据

Table 1 In vitro anti-toxoplasma activity of compounds

\begin{tabular}{|c|c|c|c|c|}
\hline Compd. & $\mathrm{R}$ & $\mathrm{CC}_{50} /\left(\mu \mathrm{mol} \cdot \mathrm{L}^{-1}\right)$ in RAW 264.7 cells & $\mathrm{IC}_{50} /\left(\mu \mathrm{mol} \cdot \mathrm{L}^{-1}\right)$ in $T$. gondii & Selectivity index \\
\hline A1 & $4-\mathrm{H}$ & 0.12 & 0.85 & 0.14 \\
\hline $\mathbf{A 2}$ & $4-\mathrm{Cl}$ & 3.25 & 5.08 & 0.64 \\
\hline $\mathbf{A 3}$ & $3,4-\mathrm{Cl}_{2}$ & 0.80 & 0.97 & 0.83 \\
\hline A4 & $4-\mathrm{Br}$ & 0.35 & 0.47 & 0.74 \\
\hline A5 & $4-\mathrm{CH}_{3}$ & 0.06 & 0.08 & 0.67 \\
\hline B1 & $4-\mathrm{H}$ & 1.14 & 1.62 & 0.70 \\
\hline B2 & $4-\mathrm{F}$ & 25.31 & 31.16 & 0.81 \\
\hline B3 & $2-\mathrm{Cl}$ & 2.67 & 3.18 & 0.84 \\
\hline B4 & $4-\mathrm{Cl}$ & 18.38 & 20.16 & 0.89 \\
\hline B5 & $2-\mathrm{Br}$ & 14.34 & 16.46 & 0.87 \\
\hline B6 & $4-\mathrm{Br}$ & 6.39 & 10.14 & 0.63 \\
\hline B7 & $4-\mathrm{OCH}_{3}$ & 0.66 & 0.84 & 0.78 \\
\hline C1 & $\mathrm{H}$ & 45.33 & 55.29 & 0.82 \\
\hline $\mathrm{C2}$ & $\mathrm{CH}_{3}$ & 32.31 & 44.00 & 0.73 \\
\hline D1 & & 435.00 & 674.00 & 0.65 \\
\hline D2 & & 647.69 & 368.50 & 1.76 \\
\hline D3 & $\begin{array}{r}\mathrm{H} \\
-\xi-\mathrm{N}\end{array}$ & 147.03 & 192.31 & 0.77 \\
\hline D4 & $\xi-\stackrel{\mathrm{H}}{\mathrm{N}}$ & 101.17 & 141.35 & 0.72 \\
\hline Chrysin & - & 54.68 & 66.37 & 0.82 \\
\hline Sulfadiazine & 一 & 1702.67 & 1865.67 & 0.91 \\
\hline
\end{tabular}

但其对宿主细胞的毒性亦有所提升. 化合物 A3 的选择 性指数略高于先导物白杨素, 表现出较强的抗弓形虫活 性. 在该系列化合物中, 其构效关系如下: $3,4-\mathrm{Cl}_{2}>4-$ $\mathrm{Br}>4-\mathrm{CH}_{3}>4-\mathrm{Cl}>\mathrm{H}$. 一定程度上表明, 苯环上引入较 多的吸电子基团其抗弓形虫活性提高. 化合物 $\mathrm{B}$ 系列引 入不同取代的茮胺基团. 其中, 化合物 B3, B4, B5 的选 择性指数优于先导物白杨素, 构效关系表明, 苯环上引 入卤素这类的吸电子基团其抗弓形虫活性优于供电子 基团. 化合物 $\mathbf{C}$ 系列引入不同的咪唑, 遗憾的是，除 $\mathbf{C 1}$ 外, 其他化合物的抗弓形虫能力与白杨素相比有所降 低. 一定程度上, 苯环与咪唑上引入供电子基团抗弓形 虫能力下降. 化合物 $\mathbf{D}$ 系列引入不同的脂肪胺类官能 团, 其中, 化合物 D2 表现出超强的抗弓形虫能力, 其 SI 值为 1.76 , 显著高于白杨素及磺胺嘧啶. 通过构效关系 推断, 很可能两个醇羟基增加了先导物的水溶性, 并且, 其氧原子作为氢键受体或者羟基的氢原子作为氢键供 体与弓形虫代谢相关酶牢固结合，抑制了其表达.

\section{2 结论}

本文通过药物化学拼合原理设计共合成了 18 个未
见报道的目标化合物. 所有目标化合物结构均经过核磁 氢谱、核磁碳谱以及高分辨质谱的确证. 目标化合物 D2 表现出最强的体外抗弓形虫活性，具备一定的新药 开发价值.

\section{3 实验部分}

\section{1 仪器与试剂}

熔点用毛细管法测定，温度计未经校正; ${ }^{1} \mathrm{H}$ NMR 和 ${ }^{13} \mathrm{C}$ NMR 使用 BRUKER AV-300 型核磁共振仪器测定 (TMS 为内标, $\mathrm{CDCl}_{3}$ 或 DMSO- $d_{6}$ 为溶剂); HRMS 数据 来源于 Thermo Scientific LTQ Orbitrap XL 型质谱仪; 本 实验所用试剂均为分析纯或化学纯.

\section{2 实验方法}

\subsection{1 中间体的合成}

中间体 1 的合成参照文献[16]方法制备，中间体 2 的合成参照文献[17]方法制备.

\subsection{2 化合物 $\mathbf{A 1} \sim \mathbf{A 5}, \mathrm{B1} \sim \mathrm{B7}, \mathrm{C1} \sim \mathrm{C2}, \mathrm{D1} \sim \mathrm{D4}$ 的合} 成通法

$50 \mathrm{~mL}$ 三口烧瓶中加入 $10 \mathrm{~mL}$ 二氯甲烷、三乙胺 
$(0.42 \mathrm{~g}, 4.2 \mathrm{mmol})$ 、不同种类的胺 $(1.4 \mathrm{mmol})$, 冰盐浴 $10{ }^{\circ} \mathrm{C}$ 下搅拌, 将中间体 $2(0.5 \mathrm{~g}, 1.4 \mathrm{mmol})$ 用适量二氯 甲烷溶解后通过恒温滴液漏斗加至反应瓶中. 薄层色谱 (TLC)监测反应, 6 10 h 反应完全, 反应液用 $10 \%$ 的盐 酸溶液调至酸性, 再加入二氯甲烷萃取, 有机相用无水 硫酸钠干燥, 抽滤, 减压旋蒸, 经硅胶色谱柱分离[展开 剂 $V$ (石油醚) $: V($ 丙酮 $)=5: 1 \sim 2: 1$ ] 得不同取代的目 标化合物 ${ }^{[17]}$.

7-甲氧基-8-( $N$-(1-苯基)-磺酰胺基)-白杨素(A1): 类白色固体, 收率 $65.3 \%$. m.p. $222 \sim 224{ }^{\circ} \mathrm{C} ;{ }^{1} \mathrm{H}$ NMR (DMSO- $\left.d_{6}, 300 \mathrm{MHz}\right) \delta: 14.00$ (s, $\left.1 \mathrm{H}, \mathrm{ArOH}\right), 10.19$ (s, $\left.1 \mathrm{H}, \mathrm{SO}_{2} \mathrm{NH}\right), 8.39 \sim 8.36(\mathrm{~m}, 2 \mathrm{H}, \mathrm{ArH}), 7.67 \sim 7.59(\mathrm{~m}$, $3 \mathrm{H}, \operatorname{ArH}), 7.26(\mathrm{~s}, 1 \mathrm{H}, \mathrm{ArH}), 7.17 \sim 7.09$ (m, 4H, ArH), $6.95(\mathrm{t}, J=6.7 \mathrm{~Hz}, 1 \mathrm{H}, \mathrm{ArH}), 6.62(\mathrm{~s}, 1 \mathrm{H}, \mathrm{ArH}), 3.97(\mathrm{~s}$, $\left.3 \mathrm{H}, \mathrm{ArOCH}_{3}\right) ;{ }^{13} \mathrm{C}$ NMR $\left(75 \mathrm{MHz}\right.$, DMSO-d $\left.d_{6}\right) \delta: 182.63$, $165.90,164.70,163.81,156.06,138.45,133.05,130.58$, 129.65 (2C), 129.54 (2C), 127.55 (2C), 124.16, 119.51 (2C), 106.38, 105.69, 104.79, 96.59, 57.97; ESI-HRMS calcd for $\mathrm{C}_{22} \mathrm{H}_{18} \mathrm{NO}_{6} \mathrm{~S}[\mathrm{M}+\mathrm{H}]^{+}$424.0849, found 424.0848 .

7-甲氧基-8-( $N$-(4-氯苯基)-磺酰胺基)-白杨素(A2): 类白色固体, 收率 $67.1 \%$. m.p. $242 \sim 244{ }^{\circ} \mathrm{C} ;{ }^{1} \mathrm{H}$ NMR $\left(\mathrm{DMSO}_{6}, 300 \mathrm{MHz}\right) \delta: 14.04(\mathrm{~s}, 1 \mathrm{H}, \mathrm{ArOH}), 10.40$ (s, $\left.1 \mathrm{H}, \mathrm{SO}_{2} \mathrm{NH}\right), 8.37(\mathrm{~d}, J=6.6 \mathrm{~Hz}, 2 \mathrm{H}, \mathrm{ArH}), 7.67 \sim 7.60$ (m, 3H, ArH), 7.28 7.24 (m, 3H, ArH), 7.16 7.13 (m, $2 \mathrm{H}, \mathrm{ArH}), 6.63$ (s, 1H, ArH), 3.97 (s, 3H, $\left.\mathrm{ArOCH}_{3}\right) ;{ }^{13} \mathrm{C}$ NMR (75 MHz, DMSO- $\left.d_{6}\right) \delta$ : 182.09, 165.54, 164.21, $163.18,155.66,136.96,132.53,130.05,129.13$ (2C), 129.05 (2C), 127.62, 127.02 (2C), 120.43 (2C), 105.51, 105.21, 104.37, 96.07, 57.50; ESI-HRMS calcd for $\mathrm{C}_{22} \mathrm{H}_{17} \mathrm{ClNO}_{6} \mathrm{~S}[\mathrm{M}+\mathrm{H}]^{+}$458.0460, found 458.0461 .

7-甲氧基-8-( $N$-(3,4-二氯)-苯基-磺酰胺基)-白杨素 (A3): 淡黄色固体, 收率 64.7\%. m.p. 238 $240{ }^{\circ} \mathrm{C} ;{ }^{1} \mathrm{H}$ NMR (DMSO- $\left.d_{6}, 300 \mathrm{MHz}\right) \delta$ : 14.05 (s, $\left.1 \mathrm{H}, \mathrm{ArOH}\right), 10.68$ (s, $\left.1 \mathrm{H}, \mathrm{SO}_{2} \mathrm{NH}\right), 8.35(\mathrm{dd}, J=6.9,0.6 \mathrm{~Hz}, 2 \mathrm{H}, \mathrm{ArH})$, $7.67 \sim 7.58$ (m, 3H, ArH), 7.44 (d, $J=9.0 \mathrm{~Hz}, 1 \mathrm{H}, \mathrm{ArH})$, $7.35(\mathrm{~d}, J=2.1 \mathrm{~Hz}, 1 \mathrm{H}, \mathrm{ArH}), 7.27$ (s, 1H, ArH), 7.10 (dd, $J=9.0,2.1 \mathrm{~Hz}, 1 \mathrm{H}, \mathrm{ArH}), 6.63$ (s, 1H, ArH), 3.96 (s, 3H, $\left.\mathrm{ArOCH}_{3}\right) ;{ }^{13} \mathrm{C}$ NMR $\left(75 \mathrm{MHz}, \mathrm{DMSO}-d_{6}\right) \delta: 182.15$, $165.82,164.29,163.34,155.78,138.36,132.62,131.32$, $131.23,130.08,129.22$ (2C), 127.05 (2C), 125.28, 119.66, 118.39, 105.38, 105.24, 104.43, 96.29, 57.63; ESI-HRMS calcd for $\mathrm{C}_{22} \mathrm{H}_{16} \mathrm{Cl}_{2} \mathrm{NO}_{6} \mathrm{~S}[\mathrm{M}+\mathrm{H}]^{+}$492.0069, found 492.0069 .

7-甲氧基-8-( $N$-(4-溴苯基)-磺酰胺基)-白杨素(A4):
淡黄色固体，收率 59.6\%. m.p. $240 \sim 242{ }^{\circ} \mathrm{C} ;{ }^{1} \mathrm{H}$ NMR (DMSO- $\left.d_{6}, 300 \mathrm{MHz}\right) \delta: 14.01(\mathrm{~s}, 1 \mathrm{H}, \mathrm{ArOH}), 10.38$ (s, $\left.1 \mathrm{H}, \mathrm{SO}_{2} \mathrm{NH}\right), 8.34(\mathrm{~d}, J=6.6 \mathrm{~Hz}, 2 \mathrm{H}, \mathrm{ArH}), 7.67 \sim 7.58$ (m, 3H, ArH), 7.36 (d, J=8.7 Hz, 2H, ArH), 7.23 (s, $1 \mathrm{H}$, ArH), 7.08 (d, $J=8.7 \mathrm{~Hz}, 2 \mathrm{H}, \operatorname{ArH}), 6.60$ (s, 1H, ArH), $3.95\left(\mathrm{~s}, 3 \mathrm{H}, \mathrm{ArOCH}_{3}\right) ;{ }^{13} \mathrm{C}$ NMR (75 MHz, DMSO- $\left.d_{6}\right) \delta$ : 182.06, 165.54, 164.24, 163.19, 155.65, 137.37, 132.53, 131.92 (2C), 130.02, 129.12 (2C), 127.00 (2C), 120.83 (2C) $115.70,105.53,105.19,104.36,96.05,57.49$; ESI-HRMS calcd for $\mathrm{C}_{22} \mathrm{H}_{17} \mathrm{BrNO}_{6} \mathrm{~S}[\mathrm{M}+\mathrm{H}]^{+}$501.9955, found 501.9954.

7-甲氧基-8-( $N$-(4-甲苯基)-磺酰胺基)-白杨素(A5): 类白色固体, 收率 $60.3 \%$. m.p. $220 \sim 222{ }^{\circ} \mathrm{C} ;{ }^{1} \mathrm{H}$ NMR (DMSO- $\left.d_{6}, 300 \mathrm{MHz}\right) \delta: 14.00$ (s, $\left.1 \mathrm{H}, \mathrm{ArOH}\right), 10.04$ (s, $\left.1 \mathrm{H}, \mathrm{SO}_{2} \mathrm{NH}\right), 8.39 \sim 8.36(\mathrm{~m}, 2 \mathrm{H}, \mathrm{ArH}), 7.67 \sim 7.60(\mathrm{~m}$, $3 \mathrm{H}, \mathrm{ArH}), 7.25$ (s, 1H, ArH), $7.05 \sim 6.95$ (m, 4H, ArH), $6.63(\mathrm{~s}, 1 \mathrm{H}, \mathrm{ArH}), 4.00\left(\mathrm{~s}, 3 \mathrm{H}, \mathrm{ArOCH}_{3}\right), 2.13(\mathrm{~s}, 3 \mathrm{H}$, $\left.\mathrm{ArOCH}_{3}\right) ;{ }^{13} \mathrm{C}$ NMR $\left(75 \mathrm{MHz}, \mathrm{DMSO}-d_{6}\right) \delta: 182.63$, $165.83,164.68,163.79,156.01,135.74,133.43,133.04$, 130.57, 129.97 (2C), 129.65 (2C), 127.54 (2C), 119.98 (2C), 106.38, 105.65, 104.78, 96.58, 57.96, 20.69; ESI-HRMS calcd for $\mathrm{C}_{23} \mathrm{H}_{20} \mathrm{NO}_{6} \mathrm{~S}[\mathrm{M}+\mathrm{H}]^{+}$438.1006, found 438.1006 .

7-甲氧基-8-( $N$-(1-芐基)-磺酰胺基)-白杨素(B1)：淡 黄色固体, 收率 57.3\%. m.p. $216 \sim 218{ }^{\circ} \mathrm{C} ;{ }^{1} \mathrm{H}$ NMR (DMSO- $\left.d_{6}, 300 \mathrm{MHz}\right) \delta: 13.92$ (s, $\left.1 \mathrm{H}, \mathrm{ArOH}\right), 8.38$ (d, $J=$ $9.0 \mathrm{~Hz}, 2 \mathrm{H}, \mathrm{ArH}), 7.98\left(\mathrm{t}, J=6.0 \mathrm{~Hz}, 1 \mathrm{H}, \mathrm{SO}_{2} \mathrm{NH}\right), 7.63 \sim$ $7.61(\mathrm{~m}, 3 \mathrm{H}, \mathrm{ArH}), 7.23(\mathrm{~s}, 1 \mathrm{H}, \mathrm{ArH}), 7.18 \sim 7.07(\mathrm{~m}, 5 \mathrm{H}$, $\mathrm{ArH}), 6.50$ (s, 1H, ArH), 4.14 (d, J=6.0 Hz, 2H, $\mathrm{NHCH}_{2}$ ), $3.94\left(\mathrm{~s}, 3 \mathrm{H}, \mathrm{ArOCH}_{3}\right) ;{ }^{13} \mathrm{C}$ NMR (75 MHz, DMSO-d $\left.d_{6}\right) \delta$ : $182.15,164.76,164.06,163.01,154.12,137.02,132.41$, 130.20, 129.07 (2C), 127.74 (2C), 127.69 (2C), 127.06 (2C), 126.89, 108.33, 104.88, 104.15, 95.87, 57.28, 46.57; ESI-HRMS calcd for $\mathrm{C}_{23} \mathrm{H}_{20} \mathrm{NO}_{6} \mathrm{~S}[\mathrm{M}+\mathrm{H}]^{+}$438.1006, found 438.1005 .

7-甲氧基-8-( $N$-(4-氟苠基)-磺酰胺基)-白杨素(B2): 类白色固体, 收率 $70.5 \%$. m.p. $236 \sim 238{ }^{\circ} \mathrm{C} ;{ }^{1} \mathrm{H}$ NMR (DMSO- $\left.d_{6}, 300 \mathrm{MHz}\right) \delta: 13.93$ (s, $\left.1 \mathrm{H}, \mathrm{ArOH}\right), 8.37$ (d, $J=$ $7.2 \mathrm{~Hz}, 2 \mathrm{H}, \mathrm{ArH}), 7.99$ (t, $\left.J=6.3 \mathrm{~Hz}, 1 \mathrm{H}, \mathrm{SO}_{2} \mathrm{NH}\right), 7.67 \sim$ 7.58 (m, 3H, ArH), 7.24 (s, 1H, ArH), 7.17 (dd, $J=8.4,5.7$ $\mathrm{Hz}, 2 \mathrm{H}, \mathrm{ArH}), 6.93$ (t, 2H, $J=9.0 \mathrm{~Hz}, \mathrm{ArH}), 6.52(\mathrm{~s}, 1 \mathrm{H}$, ArH), $4.12\left(\mathrm{~d}, J=6.3 \mathrm{~Hz}, 2 \mathrm{H}, \mathrm{NHCH}_{2}\right), 3.94(\mathrm{~s}, 3 \mathrm{H}$, $\left.\mathrm{ArOCH}_{3}\right) ;{ }^{13} \mathrm{C}$ NMR (75 MHz, DMSO- $\left.d_{6}\right) \delta: 182.11$, $164.82,163.51$ (d, $J=83.16 \mathrm{~Hz}, 2 \mathrm{C}$ ) $, 162.83,159.61$, $154.06,133.30,133.26,129.70$ (d, $J=8.16 \mathrm{~Hz}, 2 \mathrm{C}$ ), 
129.05 (2C), 127.05 (2C), 114.42 (d, $J=21.21 \mathrm{~Hz}, 2 \mathrm{C}$ ), 108.32, 104.89, 104.11, 95.84, 57.30, 45.81; ESI-HRMS calcd for $\mathrm{C}_{23} \mathrm{H}_{19} \mathrm{FNO}_{6} \mathrm{~S}[\mathrm{M}+\mathrm{H}]^{+}$456.0911, found 456.0912 .

7-甲氧基-8-( $N$-(2-氯芐基)-磺酰胺基)-白杨素(B3): 类白色固体, 收率 $64.7 \%$. m.p. 206 206 ${ }^{\circ} \mathrm{C} ;{ }^{1} \mathrm{H}$ NMR (DMSO- $\left.d_{6}, 300 \mathrm{MHz}\right) \delta: 13.94$ (s, $\left.1 \mathrm{H}, \mathrm{ArOH}\right), 8.37$ (d, $J=$ $6.3 \mathrm{~Hz}, 2 \mathrm{H}, \mathrm{ArH}), 8.06\left(\mathrm{t}, J=6.3 \mathrm{~Hz}, 1 \mathrm{H}, \mathrm{SO}_{2} \mathrm{NH}\right), 7.65 \sim$ 7.57 (m, 3H, ArH), $7.41 \sim 7.38$ (m, 1H, ArH), 7.23 (s, 1H, ArH), $7.18 \sim 7.10(\mathrm{~m}, 3 \mathrm{H}, \mathrm{ArH}), 6.47(\mathrm{~s}, 1 \mathrm{H}, \mathrm{ArH}), 4.28$ (d, $J=6.3 \mathrm{~Hz}, 2 \mathrm{H}, \mathrm{NHCH}_{2}$ ), 3.93 (s, 3H, $\left.\mathrm{ArOCH}_{3}\right) ;{ }^{13} \mathrm{C}$ NMR $\left(75 \mathrm{MHz}\right.$, DMSO- $\left.d_{6}\right) \delta$ : 182.12, 164.86, 164.10, $163.01,154.14,134.33,132.39,132.11,130.38,130.18$, 129.04 (2C), 128.83, 128.66, 127.08 (2C), 126.54, 107.91, 104.90, 104.10, 95.73, 57.20, 43.81; ESI-HRMS calcd for $\mathrm{C}_{23} \mathrm{H}_{19} \mathrm{ClNO}_{6} \mathrm{~S}[\mathrm{M}+\mathrm{H}]^{+}$472.0616, found 472.0616.

7-甲氧基-8-( $N$-(4-氯苄基)-磺酰胺基)-白杨素(B4): 淡黄色固体, 收率 $64.3 \%$. m.p. $252 \sim 254{ }^{\circ} \mathrm{C} ;{ }^{1} \mathrm{H}$ NMR (DMSO- $\left.d_{6}, 300 \mathrm{MHz}\right) \delta: 13.96$ (s, 1H, ArOH ), 8.39 8.32 (m, $2 \mathrm{H}, \mathrm{ArH}), 8.01\left(\mathrm{t}, J=6.3 \mathrm{~Hz}, 1 \mathrm{H}, \mathrm{SO}_{2} \mathrm{NH}\right), 7.67 \sim 7.57$ (m, 3H, ArH), 7.24 (s, 1H, ArH), 7.15 (s, 4H, ArH), 6.54 (s, $1 \mathrm{H}, \mathrm{ArH}), 4.13\left(\mathrm{~d}, J=6.6 \mathrm{~Hz}, 2 \mathrm{H}, \mathrm{NHCH}_{2}\right), 3.96$ (s, $3 \mathrm{H}, \mathrm{ArOCH}_{3}$ ); ${ }^{13} \mathrm{C}$ NMR (75 MHz, DMSO- $d_{6}$ ) $\delta: 182.12$, $164.91,164.02,162.97,154.02,136.02,132.40,131.74$, $130.18,129.54$ (2C), 129.07 (2C), 127.63 (2C), 127.05 (2C), 108.30, 104.90, 104.12, 95.87, 57.34, 45.87; ESIHRMS calcd for $\mathrm{C}_{23} \mathrm{H}_{19} \mathrm{ClNO}_{6} \mathrm{~S}[\mathrm{M}+\mathrm{H}]^{+} 472.0616$, found 472.0619 .

7-甲氧基-8-( $N$-(2-溴苄基)-磺酰基)-白杨素(B5)：类 白色固体, 收率 $62.9 \%$. m.p. $208 \sim 210{ }^{\circ} \mathrm{C} ;{ }^{1} \mathrm{H}$ NMR (DMSO- $\left.d_{6}, 300 \mathrm{MHz}\right) \delta: 13.95$ (s, $\left.1 \mathrm{H}, \mathrm{ArOH}\right), 8.36$ (d, $J=$ $6.3 \mathrm{~Hz}, 2 \mathrm{H}, \mathrm{ArH}), 8.07$ (t, $\left.J=6.3 \mathrm{~Hz}, 1 \mathrm{H}, \mathrm{SO}_{2} \mathrm{NH}\right), 7.66 \sim$ 7.56 (m, 3H, ArH), 7.43 (dd, J=7.5, $1.5 \mathrm{~Hz}, 1 \mathrm{H}, \mathrm{ArH})$, $7.37(\mathrm{dd}, J=7.8,0.9 \mathrm{~Hz}, 1 \mathrm{H}, \operatorname{ArH}), 7.23 \sim 7.22(\mathrm{~m}, 1 \mathrm{H}$, ArH), $7.20 \sim 7.17$ (m, 1H, ArH), 7.07 (td, $J=7.5,1.5 \mathrm{~Hz}$, $1 \mathrm{H}, \operatorname{ArH}), 5.76(\mathrm{~s}, 1 \mathrm{H}, \mathrm{ArH}), 4.27(\mathrm{~d}, J=6.3 \mathrm{~Hz}, 2 \mathrm{H}$, $\left.\mathrm{NHCH}_{2}\right), 3.94\left(\mathrm{~s}, 3 \mathrm{H}, \mathrm{ArOCH}_{3}\right) ;{ }^{13} \mathrm{C}$ NMR $(75 \mathrm{MHz}$, DMSO- $\left.d_{6}\right) \delta: 182.15,164.89,164.16,163.03,154.22$, $136.11,132.40,131.98,130.30,130.20,129.05$ (3C), $127.18,127.12$ (2C), 122.40, $107.92,104.96,104.17$, 95.80, 57.26, 46.25; ESI-HRMS calcd for $\mathrm{C}_{23} \mathrm{H}_{19} \mathrm{BrNO}_{6} \mathrm{~S}$ $[\mathrm{M}+\mathrm{H}]^{+}$516.0111, found 516.0114.

7-甲氧基-8-( $N$-(4-溴苄基)-磺酰胺基)-白杨素(B6): 淡黄色固体, 收率 72.4\%. m.p. 244 246 ${ }^{\circ} \mathrm{C} ;{ }^{1} \mathrm{H}$ NMR $\left(\mathrm{DMSO}-d_{6}, 300 \mathrm{MHz}\right) \delta: 13.96$ (s, $\left.1 \mathrm{H}, \mathrm{ArOH}\right), 8.40 \sim 8.33$ (m, 2H, ArH), 8.00 (t, $\left.J=6.6 \mathrm{~Hz}, 1 \mathrm{H}, \mathrm{SO}_{2} \mathrm{NH}\right), 7.67 \sim 7.56$ (m, 3H, ArH), 7.29 (s, 1H, ArH), 7.26 (s, 1H, ArH), 7.24 (s, $1 \mathrm{H}, \operatorname{ArH}), 7.12 \sim 7.01(\mathrm{~m}, 2 \mathrm{H}, \operatorname{ArH}), 6.54(\mathrm{~s}, 1 \mathrm{H}, \operatorname{ArH})$, $4.11\left(\mathrm{~d}, J=6.6 \mathrm{~Hz}, 2 \mathrm{H}, \mathrm{NHCH}_{2}\right), 3.96$ (s, $\left.3 \mathrm{H}, \mathrm{ArOCH}_{3}\right)$; ${ }^{13} \mathrm{C}$ NMR (75 MHz, DMSO- $d_{6}$ ) $\delta: 182.13,164.94,164.01$, $162.97,153.99,136.40,132.40,130.57$ (2C), 130.19, 129.89 (2C), 129.07 (2C), 127.05 (2C), 120.25, 108.32, 104.92, 104.13, 95.87, 57.35, 45.95; ESI-HRMS calcd for $\mathrm{C}_{23} \mathrm{H}_{19} \mathrm{BrNO}_{6} \mathrm{~S}[\mathrm{M}+\mathrm{H}]^{+}$516.0111, found 516.0111.

7-甲氧基-8-( $N$-(4-甲氧基苄基)-磺酰胺基)-白杨素 (B7): 类白色固体, 收率 $58.2 \%$. m.p. 222 $224{ }^{\circ} \mathrm{C} ;{ }^{1} \mathrm{H}$ NMR (DMSO- $\left.d_{6}, 300 \mathrm{MHz}\right) \delta: 13.92$ (d, $J=2.4 \mathrm{~Hz}, 1 \mathrm{H}$, ArOH), 8.39 (d, $J=7.8 \mathrm{~Hz}, 2 \mathrm{H}, \mathrm{ArH}), 7.83$ (t, $J=6.3 \mathrm{~Hz}$, $\left.1 \mathrm{H}, \mathrm{SO}_{2} \mathrm{NH}\right), 7.67 \sim 7.59(\mathrm{~m}, 3 \mathrm{H}, \operatorname{ArH}), 7.23$ (brs, $1 \mathrm{H}$, ArH), 6.99 (d, $J=9.0 \mathrm{~Hz}, 2 \mathrm{H}, \mathrm{ArH}), 6.57$ (dd, $J=8.7,2.1$ $\mathrm{Hz}, 2 \mathrm{H}, \mathrm{ArH}), 6.48$ (d, $J=1.8 \mathrm{~Hz}, 1 \mathrm{H}, \mathrm{ArH}), 4.08$ (d, $J=$ $\left.6.3 \mathrm{~Hz}, 2 \mathrm{H}, \mathrm{NHCH}_{2}\right), 3.95$ (s, 3H, $\left.\mathrm{ArOCH}_{3}\right), 3.61$ (d, $J=$ $\left.2.4 \mathrm{~Hz}, 3 \mathrm{H}, \mathrm{ArOCH}_{3}\right) ;{ }^{13} \mathrm{C}$ NMR (75 MHz, DMSO- $\left.d_{6}\right) \delta$ : $182.13,164.67,164.00,162.96,158.29,153.82,132.39$, $130.24,129.07$ (4C), 128.51, 127.06 (2C), 112.89 (2C), $108.68,104.83,104.10,95.83,57.29,54.86,46.27$; ESI-HRMS calcd forC ${ }_{24} \mathrm{H}_{22} \mathrm{NO}_{7} \mathrm{~S}[\mathrm{M}+\mathrm{H}]^{+}$468.1112, found 468.1113 .

7-甲氧基-8-((1H-咪唑-1-基)磺酰胺基)-白杨素(C1): 淡黄色固体, 收率 73.1\%. m.p. 200 202 ${ }^{\circ} \mathrm{C} ;{ }^{1} \mathrm{H}$ NMR (DMSO- $\left.d_{6}, 300 \mathrm{MHz}\right) \delta$ : 14.38 (s, $1 \mathrm{H}, \mathrm{ArOH}$ ), 8.35 8.29 (m, 3H, ArH), $7.67 \sim 7.60$ (m, 4H, ArH), 7.35 (s, 1H, ArH), 7.09 (d, $J=9.0 \mathrm{~Hz}, 1 \mathrm{H}, \mathrm{ArH}), 6.70$ (s, 1H, ArH), 3.90 (s, $\left.3 \mathrm{H}, \mathrm{ArOCH}_{3}\right) ;{ }^{13} \mathrm{C}$ NMR $\left(75 \mathrm{MHz}, \mathrm{DMSO}-d_{6}\right) \delta: 182.24$, $167.66,164.58,164.23,155.55,137.84,132.62,131.50$, $129.99,129.80,129.16$ (3C), 128.63, 105.60, 104.77, 103.59, 96.41, 57.71; ESI-HRMS calcd for $\mathrm{C}_{19} \mathrm{H}_{15} \mathrm{~N}_{2} \mathrm{O}_{6} \mathrm{~S}$ $[\mathrm{M}+\mathrm{H}]^{+}$399.0645, found 399.0649.

7-甲氧基-8-((2-甲基-1H-咪唑-1-基)磺酰胺基)-白杨 素(C2): 类白色固体, 收率 62.5\%. m.p. 184 186 ${ }^{\circ} \mathrm{C} ;{ }^{1} \mathrm{H}$ NMR (DMSO- $\left.d_{6}, 300 \mathrm{MHz}\right) \delta: 14.39$ (s, $1 \mathrm{H}, \mathrm{ArOH}$ ), 8.34 (dd, $J=7.5,1.2 \mathrm{~Hz}, 2 \mathrm{H}, \mathrm{ArH}), 7.67 \sim 7.60(\mathrm{~m}, 4 \mathrm{H}, \mathrm{ArH})$, 7.37 (s, 1H, ArH), 6.87 (d, J=1.8 Hz, 1H, ArH), 6.72 (s, $1 \mathrm{H}, \mathrm{ArH}), 3.89$ (s, 3H, $\left.\mathrm{ArOCH}_{3}\right), 2.39$ (s, 3H, ArH); ${ }^{13} \mathrm{C}$ NMR $\left(75 \mathrm{MHz}\right.$, DMSO- $\left.d_{6}\right) \delta$ : $182.15,167.68,164.40$, $164.09,155.48,145.20,132.65,129.95,129.22$ (2C), 126.97 (2C), 126.32, 120.73, 105.85, 104.83, 103.65, 96.56, 57.73, 14.55; ESI-HRMS calcd for $\mathrm{C}_{20} \mathrm{H}_{17} \mathrm{~N}_{2} \mathrm{O}_{6} \mathrm{~S}$ $[\mathrm{M}+\mathrm{H}]^{+}$413.0801, found 413.0804.

7-甲氧基-8-( $N, N$-二乙基-磺酰胺基)-白杨素(D1): 
淡黄色固体, 收率 53.4\%. m.p. $158 \sim 160{ }^{\circ} \mathrm{C} ;{ }^{1} \mathrm{H}$ NMR (DMSO- $\left.d_{6}, 300 \mathrm{MHz}\right) \delta: 13.99$ (s, $\left.1 \mathrm{H}, \mathrm{ArOH}\right), 8.35$ (d, $J=$ $6.6 \mathrm{~Hz}, 2 \mathrm{H}, \operatorname{ArH}), 7.67 \sim 7.58$ (m, 3H, ArH), 7.24 (s, 1H, ArH), $6.70(\mathrm{~s}, 1 \mathrm{H}, \mathrm{ArH}), 4.01$ (s, 3H, $\left.\mathrm{ArOCH}_{3}\right), 3.30 \sim$ 3.27 (m, 4H, $\mathrm{CH}_{2} \mathrm{NCH}_{2}$ ), 1.09 (t, $J=6.9 \mathrm{~Hz}, \mathrm{CH}_{2} \mathrm{CH}_{3}, 6 \mathrm{H}$, $\mathrm{CH}_{2} \mathrm{CH}_{3}$ ); ${ }^{13} \mathrm{C}$ NMR (75 MHz, DMSO- $d_{6}$ ) $\delta: 182.25$, $164.94,164.22,163.21,155.11,132.45,130.21,129.07$ (2C), 127.07 (2C), 108.48, 105.09, 104.54, 96.19, 57.31, 42.20 (2C), 14.58 (2C); ESI-HRMS calcd for $\mathrm{C}_{20} \mathrm{H}_{22} \mathrm{NO}_{6} \mathrm{~S}$ $[\mathrm{M}+\mathrm{H}]^{+}$404.1162, found 404.1163.

7-甲氧基-8-( $N, N$-双 (二乙羟基)-磺酰胺基)-白杨素 (D2): 类白色固体, 收率 62.3\%. m.p. 202 204 ${ }^{\circ} \mathrm{C} ;{ }^{1} \mathrm{H}$ NMR (DMSO- $\left.d_{6}, 300 \mathrm{MHz}\right) \delta$ : 13.99 (brs, $1 \mathrm{H}, \mathrm{ArOH}$ ), $8.35(\mathrm{~d}, J=6.3 \mathrm{~Hz}, 2 \mathrm{H}, \mathrm{ArH}), 7.64 \sim 7.59(\mathrm{~m}, 3 \mathrm{H}, \mathrm{ArH})$, $7.26(\mathrm{~s}, 1 \mathrm{H}, \operatorname{ArH}), 6.71(\mathrm{~s}, 1 \mathrm{H}, \operatorname{ArH}), 4.01(\mathrm{~s}, 3 \mathrm{H}$, $\mathrm{ArOCH}_{3}$ ), 3.89 (brs, $2 \mathrm{H}, \mathrm{CH}_{2} \mathrm{OH}, \mathrm{CH}_{2} \mathrm{OH}$ ), 3.52 (t, $J=6.0$ $\left.\mathrm{Hz}, 4 \mathrm{H}, \mathrm{CH}_{2} \mathrm{OH}, \mathrm{CH}_{2} \mathrm{OH}\right), 3.32(\mathrm{t}, J=6.0 \mathrm{~Hz}, 4 \mathrm{H}$, $\mathrm{CH}_{2} \mathrm{NCH}_{2}$ ); ${ }^{13} \mathrm{C}$ NMR (75 MHz, DMSO- $\left.d_{6}\right) \delta: 182.22$, $164.92,164.18,163.13,155.31,132.45,130.16,129.07$ (2C), 127.04 (2C), 107.95, 105.07, 104.57, 96.17, 59.94 (2C), 57.37, 51.20 (2C); ESI-HRMS calcd for $\mathrm{C}_{20} \mathrm{H}_{22} \mathrm{NO}_{8} \mathrm{~S}$ $[\mathrm{M}+\mathrm{H}]^{+}$436.1060, found 436.1064 .

7-甲氧基-8-( $\mathrm{N}$-丁基-磺酰胺基)-白杨素 (D3): 类白 色固体, 收率 57.3\%. m.p. 202 204 ${ }^{\circ} \mathrm{C} ;{ }^{1} \mathrm{H} \mathrm{NMR}\left(\mathrm{CDCl}_{3}\right.$, $300 \mathrm{MHz}) \delta: 13.93(\mathrm{~s}, 1 \mathrm{H}, \mathrm{ArOH}), 8.22 \sim 8.18(\mathrm{~m}, 2 \mathrm{H}$, ArH), $7.59 \sim 7.51(\mathrm{~m}, 3 \mathrm{H}, \mathrm{ArH}), 6.82(\mathrm{~s}, 1 \mathrm{H}, \mathrm{ArH}), 6.48(\mathrm{~s}$, $1 \mathrm{H}, \mathrm{ArH}), 5.11\left(\mathrm{t}, J=6.0 \mathrm{~Hz}, 1 \mathrm{H}, \mathrm{SO}_{2} \mathrm{NH}\right), 4.06(\mathrm{~s}, 3 \mathrm{H}$, $\left.\mathrm{ArOCH}_{3}\right), 3.01$ (q, $\left.J=6.9 \mathrm{~Hz}, 2 \mathrm{H}, \mathrm{NHCH}_{2}\right), 1.53 \sim 1.45$ (m, $2 \mathrm{H}, \mathrm{NHCH}_{2} \mathrm{CH}_{2}$ ), $1.37 \sim 1.27\left(\mathrm{~m}, 2 \mathrm{H}, \mathrm{CH}_{2} \mathrm{CH}_{3}\right), 0.88$ (t, $\left.J=7.2 \mathrm{~Hz}, 3 \mathrm{H}, \mathrm{CH}_{2} \mathrm{CH}_{3}\right) ;{ }^{13} \mathrm{C}$ NMR $(75 \mathrm{MHz}$, DMSO- $d_{6}$ ) $\delta: 182.23,164.78,164.14,163.04,154.77$, 132.41, 130.14, 129.02 (2C), 127.03 (2C), 107.68, 104.97, $104.43, \quad 96.06, \quad 57.32, \quad 42.30, \quad 30.97, \quad 19.25, \quad 13.46$; ESI-HRMS $m / z$ calcd for $\mathrm{C}_{20} \mathrm{H}_{22} \mathrm{NO}_{6} \mathrm{~S}[\mathrm{M}+\mathrm{H}]^{+} 404.1162$, found 404.1165 .

7- 甲氧基-8-( $N$-(2-羟基乙基)-磺酰胺基)-白杨素 (D4): 淡黄色固体, 收率 69.3\%. m.p. 228 230 ${ }^{\circ} \mathrm{C} ;{ }^{1} \mathrm{H}$ NMR (DMSO- $\left.d_{6}, 300 \mathrm{MHz}\right) \delta$ : 13.99 (s, $\left.1 \mathrm{H}, \mathrm{ArOH}\right)$, $8.39 \sim 8.32(\mathrm{~m}, 2 \mathrm{H}, \mathrm{ArH}), 7.67 \sim 7.57(\mathrm{~m}, 3 \mathrm{H}, \mathrm{ArH}), 7.29$ (d, $J=6.0 \mathrm{~Hz}, 1 \mathrm{H}, \operatorname{ArH}), 7.26$ (s, $1 \mathrm{H}, \mathrm{ArH}), 6.72(\mathrm{~s}, 1 \mathrm{H}$, $\mathrm{ArH}), 4.67\left(\mathrm{t}, J=5.4 \mathrm{~Hz}, 1 \mathrm{H}, \mathrm{CH}_{2} \mathrm{OH}\right.$ ), $4.02(\mathrm{~s}, 3 \mathrm{H}$, $\left.\mathrm{ArOCH}_{3}\right), 3.41\left(\mathrm{dd}, J=12.3,6.3 \mathrm{~Hz}, 2 \mathrm{H}, \mathrm{NHCH}_{2}\right), 2.92$ (dd, $\left.J=12.3,6.3 \mathrm{~Hz}, 2 \mathrm{H}, \mathrm{CH}_{2} \mathrm{OH}\right) ;{ }^{13} \mathrm{C}$ NMR $(75 \mathrm{MHz}$, DMSO- $d_{6}$ ) $\delta: 182.22,164.87,164.14,154.75,132.40$, 130.15, 129.02 (2C), 127.03 (2C), 107.44, 105.01, 104.45,
96.13, 59.63, 57.43, 45.27; ESI-HRMS calcd for $\mathrm{C}_{18} \mathrm{H}_{18^{-}}$ $\mathrm{NO}_{7} \mathrm{~S}[\mathrm{M}+\mathrm{H}]^{+} 392.0799$, found 392.0792 .

\section{3 体外抗弓形虫实验}

\subsection{1 弓形虫的复苏与传代}

弓形虫 RH 株保存于延边大学药学院细胞培养室液 氮罐内. 实验前, 从液氮罐中取出冻存管, 立即放入 $37{ }^{\circ} \mathrm{C}$ 水浴锅中快速晃动, 直至虫体悬浮液完全溶解, 经腹腔接种于雌性昆明小鼠, 3 4 d 传代 1 次, 连续传 代至毒力稳定后收集腹水中的虫体, 以细胞培养液重 悬, 备用.

\subsubsection{RAW264.7 细胞的培养}

RAW264.7 细胞为小鼠源巨噬细胞, 培养在含 $10 \%$ FBS、 $100 \mu \mathrm{g} / \mathrm{mL}$ 青霉素和 $100 \mu \mathrm{g} / \mathrm{mL}$ 链霉素的 DMEM 培养基中, 置于 $37{ }^{\circ} \mathrm{C}$, 含 $5 \% \mathrm{CO}_{2}$ 的培养箱中培养, 每 隔 2 3 d 传代 1 次.

\subsection{3 体外抗弓形虫活性的评估}

利用噻唑蓝(MTT)法收集对数生长期的 RAW264.7 细胞, 以每孔 $1 \times 10^{4}$ 个接种于 96 孔板内, 并置于 $\mathrm{CO}_{2}$ 培养箱中培养 $24 \mathrm{~h}$, 获得单细胞层; 更换细胞培养液为 含 $10 \% \mathrm{FBS}$ 的 DMEM 液, 并按照每孔细胞数：弓形虫 数 $=1: 5$ 的比例加入弓形虫速殖子, 孵育 $24 \mathrm{~h}$, 然后加 入不同浓度的受试药物 $20 \mu \mathrm{L}$, 并以磺胺嘧啶与 DMSO 分别作为阳性对照及阴性对照, 每个浓度设置 3 个平行 孔, 并设空白组. 于 $37{ }^{\circ} \mathrm{C}, 5 \% \mathrm{CO}_{2}$ 培养箱内培养 $24 \mathrm{~h}$, 每孔加入 $20 \mu \mathrm{L}$ 的 MTT 溶液, 继续睬育 $4 \mathrm{~h}$. 离心, 弃上 清液, 每孔加 DMSO $150 \mathrm{uL}$, 在酶标仪中于波长 $570 \mathrm{~nm}$ 处测定 OD 值(吸光度).

相同实验条件下测定受试药物的细胞毒性，除了细 胞未用弓形虫感染, 其他操作方法相同. 测定 OD 值后 计算各组细胞的抑制率及 $\mathrm{IC}_{50}$, 进而计算出各个药物的 选择性系数(SI).

$$
\begin{aligned}
& \text { 抑制率 }(\%)=\left(1-\frac{\mathrm{OD}_{\text {实验组 }}-\mathrm{OD}_{\text {空白组 }}}{\mathrm{OD}_{\text {对照组 }}-\mathrm{OD}_{\text {空白组 }}}\right) \times 100 \% \\
& \text { 选择性指数 }(\mathrm{SI})=\frac{\mathrm{CC}_{50}}{\mathrm{IC}_{50}}
\end{aligned}
$$

辅助材料(Supporting Information) 目标化合物的核 磁氢谱、核磁碳谱以及高分辨质谱. 这些材料可以免费 从本刊网站(http://sioc-journal.cn/)上下载.

\section{References}

[1] Rostami, A.; Riahi, S. M.; Fakhri, Y.; Saber, V; Hanifehpour, H; Valizadeh, S; Gholizadeh, M; Pouya, R. H.; Gamble, H. R. Vet. Parasitol. 2017, 244, 12.

[2] Cheng, W.; Wang, C.; Xu, T.; Liu, F.; Pappoe, F.; Luo, Q. L.; Xu, Y. H.; Lu, F. L.; Shen, J. L Vet .Parasitol. 2017, 10, 580. 
[3] Flegr, J.; Prandota, J.; Soviková, M; Israili, Z. H. Plosone 2014, 9, e90203.

[4] Hill, D. E.; Dubey, J. P. Microbiol. Spectr. 2016, 4, 1.

[5] Mui, E. J.; Jacobus, D.; Milhous, W. K.; Schiehser, G.; Hsu, H.; Roberts, C. W.; Kirisits, M. J.; McLeod, R. Antimicrob. Agents Chemother. 2005, 49, 3463.

[6] Derouin, F.; Jacqz-Aigrain, E.; Thulliez, P.; Couvreur, J.; Leport, C. Parasitol. Today 2002, 16, 254.

[7] Sak, K. Asian Pac. J. Cancer Prev. 2014, 15, 8007.

[8] Feng, X.; Qin, H.; Shi, Q.; Zhang, Y.; Zhou, F. F.; Wu, H. C.; Ding, S.; Niu, Z. Y.; Lu, Y.; Shen, P. P. Biochem. Pharmacol. 2014, 89, 503.

[9] Chan, E. C. H.; Patchareewan, P.; Owen, L.; Cardiovasc, W. J. Pharmacol. 2000, 35, 326.

[10] Lee, J. H.; Kim, Y. S.; Lee, C. K.; Lee, H. K.; Han, S.; Saengyak
Hakhoechi, S. Chem. Chemother. 1999, 30, 34.

[11] Shin, J. S.; Kim, K. S.; Kim, M. B.; Jeong, J. H.; Kim, B. K. Bioorg. Med. Chem. Lett. 1999, 9, 869.

[12] Zanoli, P.; Avallone, R.; Baraldi, M. Fitoterapia 2000, 71, 117.

[13] Adeyemi, O. S.; Sugi, T.; Han, Y. M.; Kato, K. Parasitol. Res. 2018, 117, 355 .

[14] Chio, L. C.; Bolyard, L. A.; Nasr, M. Antimicrob. Agents Chemother. 1996, 40, 727.

[15] Yeo, S. J.; Jin, C. M.; Kim, S. Y.; Park, H. Korean J. Parasitol. 2016, 54, 155.

[16] Lu, K,; Chu, J.; Wang, H. M.; Fu,X. 1; Quan, D. W.; Ding, H. X.; Yao, Q. W.; Yu, P. Tetrahedron Lett. 2013, 54, 6345.

[17] Li, Y. Q.; Zhao, W. J.; Song, Q. L.; Zhang, X. F.; Li, Z. CN $103214444,2015$. 